\title{
EVERY SYMPLECTIC MANIFOLD IS A (LINEAR) COADJOINT ORBIT
}

\author{
PAUL DONATO AND PATRICK IGLESIAS-ZEMMOUR
}

\begin{abstract}
We prove that every symplectic manifold is a coadjoint orbit of the group of automorphisms of the integration bundle of the symplectic manifold, acting linearly on its space of momenta. And that, for any group of periods of the symplectic form. This result generalizes the Kirilov-Kostant-Souriau theorem when the symplectic manifold is homogeneous under the action of a Lie group, and the symplectic form is integral.
\end{abstract}

\section{INTRODUCTION}

It is well known since Kostant, Souriau and Kirillov [Kos7o] [Sou7o] [Kir74], that a symplectic manifold $(\mathrm{X}, \omega)$, homogeneous under the action of a Lie group, is isomorphic - up to a covering - to a coadjoint orbit, possibly affine.

It is less known that any symplectic manifold ${ }^{\mathrm{I}}$ is isomorphic to a coadjoint orbit of its group of symplectomorphisms (or Hamiltonian diffeomorphisms), possibly affine [PIZi6]. This has been established, in particular, in the rigorous framework of diffeology and uses essentially the notion of Moment Map for that category [PIZıo]. But this theorem still seems to lack something. Although this is not a fundamental flaw, we would like to get rid of the affine action, defined by a twisted cocycle of the automorphisms, and prefer to identify the symplectic manifold with an ordinary coadjoint orbit, that is an orbit of the usual linear coadjoint action ${ }^{2}$. That could be done by integrating the affine cocycle in some extension of the group of automorphisms first, and then identify the affine coadjoint orbit with an ordinary coadjoint orbit of this extension. This is quite a standard procedure in ordinary differential geometry, when it is possible.

But we recall that we are no more in the classical framework but in diffeology, and we shall see that the difficulty to integrate this cocycle in an extension of the automorphisms is absorbed in this category by the ability to treat irrational toruses. The fundamental element is the integration bundle existing for any symplectic manifold, as it has been

Date: November 26, 2019.

1991 Mathematics Subject Classification. 53Do5, 58B99.

Key words and phrases. Diffeology, Symplectic Geometry, Quantization.

${ }^{I}$ We assume in the following every manifold Hausdorff and second countable.

${ }^{2}$ Thanks to François Ziegler who first suggested to make this improvement. 
established in the paper "La Trilogie du Moment" [PIZ95]. This is a principal fiber bundle over the manifold, with group the torus of periods of the symplectic form. That is, the quotient of the real line by the group of periods, i.e. the integrals of the 2 -form on every 2-cycles. This principal bundle comes equipped with a connexion form, with curvature the symplectic form. Of course the torus of periods is almost never a manifold, but still a regular diffeological group, non-trivial as a few examples have shown [PDPI83] [IZL9o]. And this is here where diffeology is inevitable. We establish first the following TheOrem I. - Let $(\mathrm{X}, \omega)$ be a symplectic manifold. Let $\mathrm{P}_{\omega}$ be its group of periods and $\mathrm{T}_{\omega}=\mathbf{R} / \mathrm{P}_{\omega}$ be its torus of periods. Let $\pi: \mathrm{Y} \rightarrow \mathrm{X}$ be an integration bundle equipped with a connexion form $\lambda$ with curvature $\omega$. Let $\operatorname{Aut}(\mathrm{Y}, \lambda)$ be the (neutral component of the) group of automorphisms of the integration structure. Then, the kernel of the projection pr: $\operatorname{Aut}(\mathrm{Y}, \lambda) \rightarrow \operatorname{Diff}(\mathrm{X}, \omega)$ is reduced to the action of the torus $\mathrm{T}_{\omega}$, and its image is the group $\operatorname{Ham}(\mathrm{X}, \omega)$ of Hamiltonian diffeomorphisms. In other words, we get an exact sequence of homomorphisms, which is a central extension:

$$
1 \rightarrow \mathrm{T}_{\omega} \rightarrow \operatorname{Aut}(\mathrm{Y}, \lambda) \rightarrow \operatorname{Ham}(\mathrm{X}, \omega) \rightarrow 1
$$

The group of Hamiltonian diffeomorphisms is defined precisely in terms of diffeology in $\left[\mathrm{PIZ}_{13}, \$_{9.15}\right.$. Then, denoting by $\mathscr{A}^{*}$ the space of momenta of the group $\operatorname{Aut}(\mathrm{Y}, \lambda)$, we prove the following theorem which reveals the universal model of symplectic manifolds.

Theorem 2. - The Moment Map $\mu_{\mathrm{Y}}: \mathrm{Y} \rightarrow \mathscr{A}^{*}$ of the action of $\operatorname{Aut}(\mathrm{Y}, \lambda)$ on $(\mathrm{Y}, d \lambda)$, is equivariant: $\mu_{\mathrm{Y}}(\varphi(y))=\operatorname{Ad}_{*}(\varphi)\left(\mu_{\mathrm{Y}}(y)\right)$ for all $\varphi \in \operatorname{Aut}(\mathrm{Y}, \lambda)$, and invariant by $\mathrm{T}_{\omega}$. Its projection $\mu_{\mathrm{X}}: \mathrm{X} \rightarrow \mathscr{A}^{*}$ is injective and caries out an identification of $\mathrm{X}$ with the orbit $\mathscr{O}_{\lambda}=\mu_{\mathrm{Y}}(\mathrm{Y})=\mu_{\mathrm{X}}(\mathrm{X})$. Therefore, every symplectic manifold is a coadjoint orbit of a linear action of a diffeological group, at least $\operatorname{Aut}(\mathrm{Y}, \lambda)$.

Note I. - The idea that every symplectic manifold is a coadjoint orbit of its group of symplectomorphisms (or Hamiltonian diffeomorphisms), is not new. It appeared already at an early age of symplectic mechanics, a few decades ago. It is mentionned for example, in a functional analysis context, by Marsden \& Weinstein in their paper on Vlasov equation [MW82, Note 3, p. 398], Taken up later by Omohundro, Weinstein's student, in his book on geometric perturbation theory in physics [Omo86, p. 364]. What was already original in the first paper [PIZi6] was the rigorous diffeology framework in which the result was proved, the role of the universal moment map, the identification of Souriau's cocycle of the action of the automorphisms and the affine action rather than linear if the cocycle is not trivial. What is original in this paper is that the affine coadjoint orbit is made linear anyway, by integrating the cocycle into a central extension of the group of Hamiltonian diffeomorphisms thanks to the integration bundle.

Note 2. - We follow the vocabulary introduced in a previous work. We call parasymplectic form any closed 2-form on a diffeological space; and a parasymplectic space any diffeological space equipped with a parasymplectic form, which we denote in general by $(\mathrm{X}, \omega)$. We refer to the textbook $\left[\mathrm{PIZ}_{\mathrm{I}}\right]$ for all generic constructions in diffeology. 


\section{Review on Diffeological Constructions}

This construction of a universal model for symplectic manifolds builds on three major constructions already established:

(I) The construction of the Moment Map for any parasymplectic form, on any diffeological space and any preserving smooth action of any diffeological group, which can be found in "Moment Map in Diffeology" [PIZıo].

(2) The general construction of the group of Hamiltonian diffeomorphisms that follows this construction op. cit. \$9.2.

(3) The integration bundle of a parasymplectic form on a manifold, in[PIZ95].

To which is added the previous work on symplectic manifolds as affine coadjoint orbits of the group of symplectomorphisms [PIZi6]. In this section we recall the basis of these main constructions.

i. The Moment Maps for Parasymplectic Spaces - First of all, let G be a diffeological group. We denote by $\mathscr{G}^{*}$ its space of its momenta, that is, the space of the left-invariant differential 1-forms on $\mathrm{G}$,

$$
\mathscr{G}^{*}=\left\{\varepsilon \in \Omega^{1}(\mathrm{G}) \mid \mathrm{L}(g)^{*}(\varepsilon)=\varepsilon, \text { for all } g \in \mathrm{G}\right\} .
$$

Now, let $(X, \omega)$ be a parasymplectic space with a parasymplectic action of $G$ on $X$. That is, a smooth morphism $\rho: G \rightarrow \operatorname{Diff}(X, \omega)$, denoted by $g \mapsto g_{X}$. Where $\operatorname{Diff}(X, \omega) \subset$ $\operatorname{Diff}(\mathrm{X})$ is the group of automorphisms of $\omega$, equipped with the functionnal diffeology. Hence, $g_{\mathrm{X}}^{*}(\omega)=\omega$ for all $g \in \mathrm{G}$.

To understand the essential nature of the moment map, which is a map from $\mathrm{X}$ to $\mathscr{G}^{*}$, it is good to consider the simplest case, and use it then as a guide to extend this simple construction to the general case.

The Simplest Case. Consider the case where $\mathrm{X}$ is a manifold, and $\mathrm{G}$ is a Lie group. Let us assume that $\omega$ is exact $\omega=d \alpha$, and that $\alpha$ is also invariant by $\mathrm{G}$. Regarding $\omega$, the moment map ${ }^{3}$ of the action of $\mathrm{G}$ on $\mathrm{X}$ is the map

$$
\mu: \mathrm{X} \rightarrow \mathscr{G}^{*} \quad \text { defined by } \quad \mu(x)=\hat{x}^{*}(\alpha),
$$

where $\hat{x}: \mathrm{G} \rightarrow \mathrm{X}$ is the orbit map $\hat{x}(g)=g_{\mathrm{X}}(x)$.

As we can see, there is no obstacle, in this simple situation, to generalize, mutatis mutandis, the Moment Map to a diffeological group acting by symmetries on a diffeological parasymplectic space. But, as we know, not all closed 2 -forms are exact, and even if they are exact, they do not necessarily have an invariant primitive. We shall see now, how we can generally come to a situation, so close to the simple case above, that modulo some minor subtleties we can build a good Moment Map in all cases.

\footnotetext{
3 Precisely, one moment map, since they are defined up to a constant.
} 
The General Case. We consider a connected parasymplectic diffeological space (X, $\omega)$, and a diffeological group $\mathrm{G}$ acting on $\mathrm{X}$ and preserving $\omega$. Let $\mathscr{K}$ be the Chain-Homotopy Operator, defined in $\left[\mathrm{PIZ}_{13}, \$ 6.83\right]$. We recall that

$$
\mathscr{K}: \Omega^{k}(\mathrm{X}) \rightarrow \Omega^{k-1}(\operatorname{Paths}(\mathrm{X}))
$$

is a linear operator which satisfies the property

$$
d \circ \mathscr{K}+\mathscr{K} \circ d=\hat{1}^{*}-\hat{0}^{*},
$$

where $\hat{t}(\gamma)=\gamma(t)$, with $t \in \mathbf{R}$ and $\gamma \in \operatorname{Paths}(\mathrm{X})$. Then, the differential 1-form $\mathscr{K} \omega$, defined on Paths $(\mathrm{X})$, is related to $\omega$ by $d[\mathscr{K} \omega]=\left(\hat{1}^{*}-\hat{0}^{*}\right)(\omega)$, and $\mathscr{K} \omega$ is invariant by $\mathrm{G}$ (op. cit. \$6.84). Considering $\bar{\omega}=\left(\hat{1}^{*}-\hat{0}^{*}\right)(\omega)$ and $\bar{\alpha}=\mathscr{K} \omega$, we are in the simple case: $\bar{\omega}=d \bar{\alpha}$ with $\bar{\alpha}$ invariant. We can apply the construction above and define then the Moment Map of Paths by

$$
\Psi: \operatorname{Paths}(\mathrm{X}) \rightarrow \mathscr{G}^{*} \quad \text { with } \Psi(\gamma)=\hat{\gamma}^{*}(\mathscr{K} \omega),
$$

and $\hat{\gamma}: G \rightarrow \operatorname{Paths}(X)$ is the orbit map $\hat{\gamma}(g)=g_{X} \circ \gamma$ of a path $\gamma$. The moment of paths is additive with respect to the concatenation,

$$
\Psi\left(\gamma \vee \gamma^{\prime}\right)=\Psi(\gamma)+\Psi\left(\gamma^{\prime}\right) .
$$

This paths Moment Map $\Psi$ is equivariant by $\mathrm{G}$, acting by composition on Paths $(\mathrm{X})$, and by coadjoint action on $\mathscr{G}^{*}$. Next, defining the Holonomy of the action of $\mathrm{G}$ on $\mathrm{X}$ by

$$
\Gamma=\{\Psi(\ell) \mid \ell \in \operatorname{Loops}(\mathrm{X})\} \subset \mathscr{G}^{*},
$$

the Two-Points Moment Map is defined by pushing $\Psi$ forward on $\mathrm{X} \times \mathrm{X}$,

$$
\psi\left(x, x^{\prime}\right)=\operatorname{class}(\Psi(\gamma)) \in \mathscr{G}^{*} / \Gamma,
$$

where $\gamma$ is a path connecting $x$ to $x^{\prime}$, and where class denotes the projection from $\mathscr{G}^{*}$ onto its quotient $\mathscr{G}^{*} / \Gamma$. The holonomy $\Gamma$ is the obstruction for the action of $\mathrm{G}$ to be Hamiltonian. The additivity of $\Psi$ becomes the Chasles' cocycle condition

$$
\psi\left(x, x^{\prime}\right)+\psi\left(x^{\prime}, x^{\prime \prime}\right)=\psi\left(x, x^{\prime \prime}\right) .
$$

Let $\mathrm{Ad}: \mathrm{G} \rightarrow \operatorname{Diff}(\mathrm{G})$ be the adjoint action, $\operatorname{Ad}(g): k \mapsto g k g^{-1}$. That induces on $\mathscr{G}^{*}$ a linear coadjoint action

$$
\operatorname{Ad}_{*}: \mathrm{G} \rightarrow \mathrm{L}\left(\mathscr{G}^{*}\right) \quad \text { with } \quad \operatorname{Ad}_{*}(g): \varepsilon \mapsto \operatorname{Ad}(g)_{*}(\varepsilon)=\operatorname{Ad}\left(g^{-1}\right)^{*}(\varepsilon) .
$$

Next, the group $\Gamma$ is made of closed forms, invariant by the linear coadjoint action. Thus, the coadjoint action passes to the quotient $\mathscr{G}^{*} / \Gamma$, and we denote the quotient action the same way:

$$
\operatorname{Ad}_{*}(g): \operatorname{class}(\varepsilon) \mapsto \operatorname{class}\left(\operatorname{Ad}_{*}(g)(\varepsilon)\right) .
$$

The 2-points Moment Map $\psi$ is equivariant for the quotient coadjoint action. Note that the quotient $\mathscr{G}^{*} / \Gamma$ is a legit diffeological Abelian group ${ }^{4}$

\footnotetext{
${ }^{4}$ For the quotient of the functional diffeology of $\mathscr{G}^{*} \subset \Omega^{1}(\mathrm{G})$ by $\Gamma$. In particular, for Lie groups, it is always a product $\mathbf{R}^{k} \times \mathrm{T}^{\ell}, k, \ell \in \mathbf{N}$.
} 
Now, because $\mathrm{X}$ is connected, there always exists a map

$$
\mu: \mathrm{X} \rightarrow \mathscr{G}^{*} / \Gamma \quad \text { such that } \quad \psi\left(x, x^{\prime}\right)=\mu\left(x^{\prime}\right)-\mu(x) .
$$

The solutions of this equation are given by

$$
\mu(x)=\psi\left(x_{0}, x\right)+c,
$$

where $x_{0} \in \mathrm{X}$ is an arbitrary point and $c \in \mathscr{G}^{*} / \Gamma$ is any constant. But this map is a priori no longer equivariant with respect to $\mathrm{Ad}_{*}$ on $\mathscr{G}^{*} / \Gamma$. Its variance introduces a 1-cocycle $\theta$ of $\mathrm{G}$ with values in $\mathscr{G}^{*} / \Gamma$ such that

$$
\mu(g(x))=\operatorname{Ad}_{*}(g)(\mu(x))+\theta(g),
$$

with

$$
\theta(g)=\psi\left(x_{0}, g\left(x_{0}\right)\right)-\Delta c(g), \quad \text { and } \quad \Delta(c): g \mapsto \operatorname{Ad}_{*}(g)(c)-c
$$

is the coboundary due to the constant $c$ in the choice of $\mu$. The cocycle $\theta$ defines then a new action of $\mathrm{G}$ on $\mathscr{G}^{*} / \Gamma$, that is, a quotient affine action :

$$
\operatorname{Ad}_{*}^{\theta}(g): \tau \mapsto \operatorname{Ad}_{*}(g)(\tau)+\theta(g) \quad \text { for all } \quad \tau \in \mathscr{G}^{*} / \Gamma .
$$

The Moment Map $\mu$ is then equivariant with respect to this affine action:

$$
\mu(g(x))=\operatorname{Ad}_{*}^{\theta}(g)(\mu(x)) .
$$

Note that, in particular, if $\mathrm{G}$ is transitive on $\mathrm{X}$, then the image of the Moment Map $\mu$ is an affine coadjoint orbit in $\mathscr{G}^{*} / \Gamma$.

This construction extends to the category \{Diffeology\}, the Moment Map for $\{$ Manifolds\} introduced by Souriau in the sixties [Sou7o].

The group of all automorphisms of a parasymplectic space is denoted by $\operatorname{Diff}(\mathrm{X}, \omega)$ or by $\mathrm{G}_{\omega}$, it is a legitimate diffeological group. The constructions above give the space of momenta $\mathscr{G}_{\omega}^{*}$, the universal path moment map $\Psi_{\omega}$, the universal bolonomy $\Gamma_{\omega}$, the universal two-points moment map $\psi_{\omega}$, the universal moment maps $\mu_{\omega}$, and the universal Souriau's cocycles $\theta_{\omega}$.

2. The Case of a Symplectic Manifold - Let $(\mathrm{X}, \omega)$ be a connected parasymplectic manifold. The value of the paths Moment Map $\Psi_{\omega}$ at the point $p \in \operatorname{Paths}(\mathrm{X})=$ $\mathrm{C}^{\infty}(\mathbf{R}, \mathrm{X})$, evaluated on the $n$-plot $\mathrm{F}: \mathrm{U} \rightarrow \mathrm{G}_{\omega}$ is explicitely given by

$$
\Psi_{\omega}(p)(\mathrm{F})_{r}(\delta r)=\int_{0}^{1} \omega_{p(t)}(\dot{p}(t), \delta p(t)) d t
$$

where $r \in \mathrm{U}$ and $\delta r \in \mathbf{R}^{n}$, $\delta p$ denotes the lifting in the tangent space TX of the path $p$, defined by

$$
\delta p(t)=[\mathrm{D}(\mathrm{F}(r))(p(t))]^{-1} \frac{\partial \mathrm{F}(r)(p(t))}{\partial r}(\delta r) \text { for all } t \in \mathbf{R} .
$$

In that case we have the foolowing theorem, see [PIZI6] for example: 
Theorem (P.I-Z). Let X be a connected Hausdorff manifold. A parasymplectic form $\omega$ on $\mathrm{X}$ is symplectic if and only if the following two conditions are satisfied:

(I) The manifold $\mathrm{X}$ is homogeneous under the action of $\mathrm{G}_{\omega}$.

(2) The universal Moment Map $\mu_{\omega}: \mathrm{X} \rightarrow \mathscr{G}_{\omega}^{*} / \Gamma_{\omega}$ is injective.

Hence, the Moment Map identifies the manifold $\mathrm{X}$ with a, a priori affine, $\left(\Gamma_{\omega}, \theta_{\omega}\right)$ coadjoint orbit $\mathscr{O}_{\omega}$ of $\mathrm{G}_{\omega}$,

$$
\mu_{\omega}(\mathrm{X})=\mathscr{O}_{\omega} \subset \mathscr{G}_{\omega}^{*} / \Gamma_{\omega} .
$$

The situation is summarized by the diagram

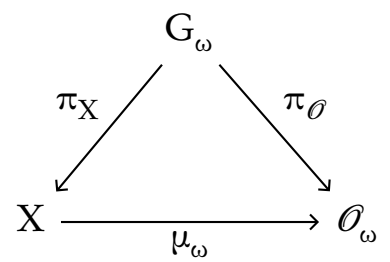

where $\pi_{\mathrm{X}}(\varphi)=\varphi\left(x_{0}\right), \pi_{\mathscr{O}}(\varphi)=\operatorname{Ad}_{*}^{\theta}(\varphi)\left(\mu_{\omega}\left(x_{0}\right)\right)$, for all $\varphi \in \mathrm{G}_{\omega}$ and $x_{0} \in \mathrm{X}$ is any base point. The projections $\pi_{X}$ is a subduction [Boo69, Don84], $\mathscr{O}_{\omega}$ is equipped with the pushforward diffeology of $\mathrm{G}_{\omega}$ by $\pi_{\mathscr{O}}$, and $\mu_{\omega}$ is then a diffeomorphism.

3. Hamiltonian Diffeomorphisms - In the construction of the Moment Map of a parasymplectic action of a diffeological group $G$ on $(X, \omega)$, the holonomy group $\Gamma$ is the obstruction of the action of $\mathrm{G}$ to be Hamiltonian.

Definition. A parasymplectic action of a diffeological group $\mathrm{G}$ on $(\mathrm{X}, \omega)$ is said to be Hamiltonian if $\Gamma=\{0\}$.

Hence, the moment maps have their values in $\mathscr{G}^{*}$. We get then the following theorem [PIZIO, \$9.2]

Theorem (P.I-Z). Let $(\mathrm{X}, \omega)$ be a connected parasymplectic diffeological space. There exists a largest connected subgroup $\operatorname{Ham}(\mathrm{X}, \omega) \subset \operatorname{Diff}(\mathrm{X}, \omega)$ whose action is Hamiltonian, that is, whose holonomy is trivial. The elements of $\operatorname{Ham}(\mathrm{X}, \omega)$ are called Hamiltonian diffeomorphisms. An action $\rho$ of a diffeological group $\mathrm{G}$ on $\mathrm{X}$ is Hamiltonian if and only if, restricted to the identity component of $\mathrm{G}, \rho$ takes its values in $\operatorname{Ham}(\mathrm{X}, \omega)$.

The group $\operatorname{Ham}(\mathrm{X}, \omega)$ is precisely built as follows. Let $\mathrm{G}_{\omega}^{\circ}$ be the neutral component of $G_{\omega}=\operatorname{Diff}(X, \omega)$. Let $\pi: \widetilde{G}_{\omega}^{\circ} \rightarrow G_{\omega}^{\circ}$ be the universal covering. Since the universal holonomy $\Gamma_{\omega}$ is made of closed momenta [PIZıo, $\$ 4.7$ ], every $\gamma \in \Gamma_{\omega}$ defines a unique homomorphism $\mathbf{k}(\gamma)$ from $\widetilde{\mathrm{G}}_{\omega}^{\circ}$ to $\mathbf{R}$ such that $\pi^{*}(\gamma)=d[\mathbf{k}(\gamma)]$ [PIZIo, \$3.II]. Let

$$
\widehat{\mathbf{H}}_{\omega}=\bigcap_{\gamma \in \Gamma_{\omega}} \operatorname{ker}(\mathbf{k}(\gamma)) \text {, then } \operatorname{Ham}(\mathrm{X}, \omega)=\pi\left(\widehat{\mathbf{H}}_{\omega}^{\circ}\right) \text {, }
$$

where $\widehat{\mathbf{H}}_{\omega}^{\circ} \subset \mathbf{H}_{\omega}$ is its neutral component. The space of momenta and the universal moment maps objects associated to $\mathrm{H}_{\omega}=\operatorname{Ham}(\mathrm{X}, \omega)$ are denoted by: $\mathscr{H}_{\omega}^{*}, \bar{\Psi}_{\omega}, \bar{\psi}_{\omega}, \bar{\mu}_{\omega}$, and $\bar{\theta}_{\omega}$. 
4. The Integration Bundle of a Parasymplectic Form - Let $(\mathrm{X}, \omega)$ be a parasymplectic manifold, connected, Hausdorff and second countable. Let $\mathrm{P}_{\omega}$ its group of periods, that is,

$$
\mathrm{P}_{\omega}=\left\{\int_{\sigma} \omega \mid \sigma \in \mathrm{H}_{2}(\mathrm{X}, \mathbf{Z})\right\} \subset \mathbf{R} .
$$

Since $\mathrm{X}$ is second countable $\mathrm{P}_{\omega}$ is diffeogicaly discrete, that is, the diffeology induced by the standard diffeology of $\mathbf{R}$ is the discrete diffeology. The plots are locally constant. Let

$$
\mathrm{T}_{\omega}=\mathbf{R} / \mathrm{P}_{\omega}
$$

be its torus of periods. Except in the case where the group of periods has only one generator, the torus of periods is not a manifold but a legitimate non trivial diffeological group. See for example the paper on the irrational torus $\mathrm{T}_{\alpha}=\mathbf{R} / \mathbf{Z}+\alpha \mathbf{Z}[\mathrm{PDPI} 83]$, where $\alpha \notin \mathbf{Q}$, for an example of such irrational torus.

Then, we get the following theorem in [PIZ95, Theorem 1.5].

THeOREM (P.I-Z). Let (X, $\omega)$ be a second countable Hausdorff parasymplectic manifold. There exists always a $\mathrm{T}_{\omega}$-principal fiber bundle $\pi: \mathrm{Y} \rightarrow \mathrm{X}$ equipped with a connexion 1-form $\lambda$ with curvature $\omega$. That is, $\pi^{*}(\omega)=d \lambda$. The various such integration bundles are classified by the extension group $\operatorname{Ext}\left(\mathrm{H}_{1}(\mathrm{X}, \mathrm{Z}), \mathrm{P}_{\omega}\right)$.

We may need to precise that a connexion 1-form $\lambda$ on $\mathrm{Y}$ is a $\mathrm{T}_{\omega}$-invariant 1-form, such that, for all $y \in \mathrm{Y}, \hat{y}^{*}(\lambda)=\theta$, where $\hat{y}: \mathrm{T}_{\omega} \rightarrow \mathrm{Y}$ is the orbit map $\hat{y}(\tau)=\tau_{\mathrm{Y}}(y)$; and $\theta$ is the canonical 1 -form on $\mathrm{T}_{\omega}$ defined by $\operatorname{class}^{*}(\theta)=d t$, with class: $\mathbf{R} \rightarrow \mathrm{T}_{\omega}$ the projection.

\section{Automorphisms Exact Sequence}

For a symplectic manifold, the transition from an affine orbit to a linear orbit ${ }^{5}$ needs to absorb the Souriau's cocycle somewhere. We do it by building an extension of the group of Hamiltonian diffeomorphisms, associated with the the integration bundle of the symplectic form $(\$ 4)$.

5. The Central Extension of Hamiltonian Diffeomorphisms - Let $(\mathrm{X}, \omega)$ be a symplectic manifold. Let $\mathrm{P}_{\omega}$ be its group of periods and $\mathrm{T}_{\omega}=\mathbf{R} / \mathrm{P}_{\omega}$ be its torus of periods. Let $\pi: \mathrm{Y} \rightarrow \mathrm{X}$ be its $\mathrm{T}_{\omega}$-principal integration fiber bundle, and $\lambda$ be its connection form. Let $\operatorname{Aut}(\mathrm{Y}, \lambda)$ be the group of automorphisms of the integration structure, that is:

$$
\operatorname{Aut}(\mathrm{Y}, \lambda)=\left\{\varphi \in \operatorname{Diff}(\mathrm{Y}) \mid \varphi^{*}(\lambda)=\lambda \text { and } \exists f \in \operatorname{Diff}(\mathrm{X}), \pi \circ \varphi=f \circ \pi\right\}
$$

Actually we reduce $\operatorname{Aut}(\mathrm{Y}, \lambda)$ to its the neutral component. Then, the diffeomorphism $f$ belongs naturally to the group of Hamiltonian diffeomorphisms $\operatorname{Ham}(\mathrm{X}, \omega)$. The

5We recall that we say "linear orbit" as a shortcut for "orbit of a linear action". 
mapping $\eta: \varphi \mapsto f$ is then a surjective homomorphism. Its kernel is the torus of periods $\mathrm{T}_{\omega}$, and $\eta$ is a central extension. Which is summarized by the exact sequence:

$$
1 \rightarrow \mathrm{T}_{\omega} \rightarrow \operatorname{Aut}(\mathrm{Y}, \lambda) \stackrel{\eta}{\rightarrow} \operatorname{Ham}(\mathrm{X}, \omega) \rightarrow 1
$$

Note. - The integration bundle of a parasymplectic form is not necessarily unique. They are all classified by $\operatorname{Ext}\left(\mathrm{H}_{1}(\mathrm{X}, \mathrm{Z}), \mathrm{P}_{\omega}\right)$ [PIZ95]. The theorem above applies to any of them indifferently, which had been noticed by Bertram Kostant and Jean-Marie Souriau in the integral case $\mathrm{P}_{\omega}=a \mathbf{Z}$. And by the way, this extension is called the Kostant-Souriau Extension in that case.

It is notable too, that all this construction is purely diffeologial, involves only differential forms and do not need tangent vectors or integration of vector fields. That aspect of diffeology had been already underlined in the construction of the Moment Map in particular in [PIZıo].

Proof. Let us begin by fixing our notation. The action of an element $\tau \in \mathrm{T}_{\omega}$ on $y \in \mathrm{Y}$ will be noted indifferently by

$$
\tau \cdot y \text { or by } \tau_{\mathrm{Y}}(y) \text {. }
$$

Now, let $\varphi \in \operatorname{Aut}(\mathrm{Y}, \lambda)$ and $f=\eta(\varphi)$. Since $f \circ \pi=\pi \circ \varphi, \varphi^{*}(\lambda)=\lambda$ and $\pi^{*}(\omega)=d \lambda$, $\pi$ being a subduction, we get $f \in \operatorname{Diff}(\mathrm{X}, \omega)$.

(A) Let us to prove that $\operatorname{ker}(\eta)=\mathrm{T}_{\omega}$, acting on $\mathrm{Y}$ by $\tau: y \mapsto \tau \cdot y$. Let $\varphi \in \operatorname{ker}(\eta)$, that is, $\pi \circ \varphi=\pi$. Then, for all $y \in \mathrm{Y}$, there exists a unique $\tau(y) \in \mathrm{T}_{\omega}$ such that $\varphi(y)=\tau(y) \cdot y$.

(a) Let us first check that $\tau: \mathrm{Y} \rightarrow \mathrm{T}_{\omega}$ is smooth. Let $r \mapsto y_{r}$ by a plot in $\mathrm{Y}$, composed by $\varphi$, we get the plot $r \mapsto \tau\left(y_{r}\right) \cdot y_{r}$. We need to prove that $r \mapsto \tau\left(y_{r}\right)$ itself is smooth. The pullback of $\pi: \mathrm{Y} \rightarrow \mathrm{X}$ by the plot $r \mapsto x_{r}=\pi\left(y_{r}\right)$ is localy trivial, then we can restrict these plots to a ball $\mathrm{B}$ over wich the pullback

$$
\left[r \mapsto x_{r}\right]^{*}(\mathrm{Y})=\left\{(r, y) \in \mathrm{B} \times \mathrm{Y} \mid \pi(y)=x_{r}\right\}
$$

is trivial. Any $\mathrm{T}_{\omega}$-principal bundle isomorphism $\mathrm{F}$ from this pullback to the product $\mathrm{B} \times \mathrm{T}_{\omega}$ writes $\mathrm{F}(r, y)=(r, t(r)(y))$, and the smooth map $t$ with values in $\mathrm{T}_{\omega}$ satisfies the equivariance $t(r)(\tau \cdot y)=\tau \cdot t(r)(y)$. Thus, $r \mapsto t(r)\left(y_{r}\right)$ is smooth as well as $r \mapsto t(r)\left(\tau\left(y_{r}\right)\left(y_{r}\right)\right)=\tau\left(y_{r}\right) \cdot t(r)\left(y_{r}\right)$. Hence, $r \mapsto \tau\left(y_{r}\right)$ is smooth. Therefore, the function $\tau$ is smooth.

(b) Let us prove now that the function $\tau$ is constant. The invariance $\varphi^{*}(\lambda)=\lambda$ implies $\lambda\left(r \mapsto \tau\left(y_{r}\right) \cdot y_{r}\right)=\lambda\left(r \mapsto y_{r}\right)$, for all plots $r \rightarrow y_{r}$. That is, thanks to the partial derivatives formula [ $\mathrm{PIZI}_{3}, \$ 8.37$ \%]

$$
\begin{aligned}
\lambda\left(r \mapsto y_{r}\right) & =\lambda\left(r \mapsto \tau\left(y_{r}\right) \cdot y_{r}\right) \\
& =\lambda\left(r \mapsto y_{r}\right)+\tau^{*}(\theta)\left(r \mapsto y_{r}\right),
\end{aligned}
$$

where $\theta$ is the canonical 1 -form on $\mathrm{T}_{\omega}$. Thus, $\tau^{*}(\theta)=0$. Then $\tau$ is constant. Hence, $\operatorname{ker} \eta=\mathrm{T}_{\omega}$. 
(B) Let us prove that $\eta$ takes its value in $\operatorname{Ham}(\mathrm{X}, \omega)$. That is, that the holonomy group of $\operatorname{Aut}(\mathrm{Y}, \lambda)$ vanishes when acting on $(\mathrm{X}, \omega)$ through the action

$$
\varphi_{\mathrm{X}}(x)=f(x) \text { with } f=\eta(\varphi) .
$$

We shall denote by $\mathscr{A}^{*}$ the space of momenta of $\operatorname{Aut}(\mathrm{Y}, \lambda)$. The Moment Map $\Psi_{\mathrm{X}}$ of the action of $\operatorname{Aut}(\mathrm{Y}, \lambda)$ on $(\mathrm{X}, \omega)$ is given, according to previous notations by:

$$
\Psi_{\mathrm{X}}: \operatorname{Paths}(\mathrm{X}) \rightarrow \mathscr{A}^{*} \text { with } \Psi(\gamma)=\hat{\gamma}^{*}\left(\mathscr{K}_{\mathrm{X}}(\omega)\right)
$$

where $\hat{\gamma}: \varphi \mapsto f \circ \gamma$ is the orbit map. Let us prove now that $\Psi_{\mathrm{X}}(\ell)=\hat{\ell}^{*}\left(\mathscr{K}_{\mathrm{X}}(\omega)\right)=0$, for all $\ell \in \operatorname{Loops}(\mathrm{X})$.

Let us recall first of all that the principal fiber bundle $\pi: Y \rightarrow X$ induces, in particular, a subduction of loops spaces:

$$
\pi_{*}: \operatorname{Loops}(\mathrm{Y}) \rightarrow \operatorname{Loops}(\mathrm{X}) \text { by pushforward } \pi_{*}(\underline{\ell})=\pi \circ \underline{\ell},
$$

see $\left[\mathrm{PIZI}_{3}, \$ 8.32\right]$ and [PIZı9]. That is, every plot $r \mapsto \ell_{r}$ in Loops $(\mathrm{X})$ has a local smooth lifting $r \mapsto \underline{\ell}_{r}$, everywhere, in Loops(Y). Note that we shall underline the paths in $Y$, to distinguish them from paths in $X$. Now, let $\ell$ and $\underline{\ell}$ such that $\pi \circ \underline{\ell}=\ell$. We have $\hat{\ell}(\varphi)=f \circ \ell=f \circ \pi \circ \underline{\ell}=\pi \circ \varphi \circ \underline{\ell}=\pi \circ \underline{\hat{\ell}}(\varphi)$, that is, $\hat{\ell}=\pi_{*} \circ \underline{\hat{\ell}}$. Thus,

$$
\hat{\ell}^{*}\left(\mathscr{K}_{\mathrm{X}}(\omega)\right)=(\pi \circ \underline{\hat{\ell}})^{*}\left(\mathscr{K}_{\mathrm{X}}(\omega)\right)=\underline{\ell}^{*}\left(\left(\pi_{*}\right)^{*}\left(\mathscr{K}_{\mathrm{X}}(\omega)\right)\right)
$$

Then, let us recall the variance of the chain-homotopy operators $\mathscr{K}_{\mathrm{X}}$ and $\mathscr{K}_{\mathrm{Y}}$, relatives to $\mathrm{X}$ and $\mathrm{Y}\left[\mathrm{PIZI}_{3}, \$ 6.84\right]$, summarized by the commutative diagram:

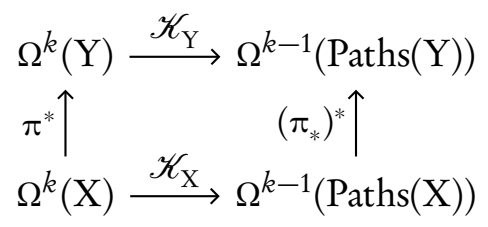

We have then:

$$
\left(\pi_{*}\right)^{*}\left(\mathscr{K}_{\mathrm{X}}(\omega)\right)=\mathscr{K}_{\mathrm{Y}}\left(\pi^{*}(\omega)\right)=\mathscr{K}_{\mathrm{Y}}(d \lambda) .
$$

Hence:

$$
\underline{\ell}^{*}\left(\left(\pi_{*}\right)^{*} \mathscr{K}_{\mathrm{X}}(\omega)\right)=\underline{\hat{\ell}}^{*}\left(\mathscr{K}_{\mathrm{Y}}(d \lambda)\right)
$$

Thus:

$$
\Psi_{\mathrm{X}}(\ell)=\hat{\ell}^{*}\left(\mathscr{K}_{\mathrm{X}}(\omega)\right)=\underline{\hat{\ell}}^{*}\left(\mathscr{K}_{\mathrm{Y}}(d \lambda)\right)=\Psi_{\mathrm{Y}}(\underline{\ell}),
$$

where $\Psi_{\mathrm{Y}}$ is the Moment Map for the action of $\operatorname{Aut}(\mathrm{Y}, d \lambda)$ acting on $(\mathrm{Y}, d \lambda)$. Note that, that could have been deduced directly from $\left[\mathrm{PIZI}_{3}, \$ 9.13\right]$. Now, according to the 
fundamental property of the Chain-Homotopy Operator, we have:

$$
\begin{aligned}
\underline{\hat{\ell}}^{*}\left(\mathscr{K}_{\mathrm{Y}}(d \lambda)\right)+\underline{\hat{\ell}}^{*}\left(d\left(\mathscr{K}_{\mathrm{Y}}(\lambda)\right)\right) & =\underline{\hat{\ell}}^{*}\left(\hat{1}^{*}(\lambda)-\hat{0}^{*}(\lambda)\right) \\
& =(\hat{1} \circ \underline{\hat{\ell}})^{*}(\lambda)-(\hat{0} \circ \underline{\hat{\ell}})^{*}(\lambda) \\
& =0
\end{aligned}
$$

because $\underline{\ell}$ is a loop. Therefore,

$$
\underline{\hat{\ell}}^{*}\left(\mathscr{K}_{\mathrm{Y}}(d \lambda)\right)=-d\left(\underline{\hat{\ell}}^{*}\left(\mathscr{K}_{\mathrm{Y}}(\lambda)\right)\right)
$$

But, for every plot $r \mapsto \varphi_{r}$ in $\operatorname{Aut}(\mathrm{Y}, \lambda)$, for all $r$ in its domain :

$$
\underline{\ell}^{*}\left(\mathscr{K}_{\mathrm{Y}}(\lambda)\right)\left(\varphi_{r}\right)=\mathscr{K}_{\mathrm{Y}}(\lambda)\left(\varphi_{r} \circ \underline{\ell}\right)=\int_{\varphi_{r *}(\underline{\ell})} \lambda=\int_{\underline{\ell}} \varphi_{r}^{*}(\lambda)=\int_{\underline{\ell}} \lambda .
$$

Hence $\underline{\ell}^{*}\left(\mathscr{K}_{\mathrm{Y}}(\lambda)\right)$ is constant, its derivative vanishes and therefore

$$
\Psi_{\mathrm{X}}(\ell)=\hat{\ell}^{*}\left(\mathscr{K}_{\mathrm{X}}(\omega)\right)=\underline{\hat{\ell}}^{*}\left(\mathscr{K}_{\mathrm{Y}}(d \lambda)\right)=0 .
$$

And that achieves to prove that $\eta: \operatorname{Aut}(\mathrm{Y}, \lambda) \rightarrow \operatorname{Diff}(\mathrm{X}, \omega)$ takes its values in $\operatorname{Ham}(\mathrm{X}, \omega)$.

(C) Let us show now that $\mathrm{T}_{\omega} \subset \operatorname{Aut}(\mathrm{Y}, \lambda)$ is central, that is, $\eta$ : $\operatorname{Aut}(\mathrm{Y}, \lambda) \rightarrow \operatorname{Ham}(\mathrm{X}, \omega)$ is a central extension. Let $\varphi \in \operatorname{Aut}(\mathrm{Y}, \lambda)$. We have seen that $\mathrm{T}_{\omega}=\operatorname{ker}(\eta)$. Thus, for all $\tau \in \mathrm{T}_{\omega}$ there exists $\tau^{\prime} \in \mathrm{T}_{\omega}$ such that $\tau^{\prime}=\varphi \circ \tau \circ \varphi^{-1}$. Obviously, $h_{\varphi}: \tau \mapsto \tau^{\prime}$ defines a group isomorphism of $\mathrm{T}_{\omega}: h_{\varphi}\left(\tau_{1} \tau_{2}\right)=h_{\varphi}\left(\tau_{1}\right) h_{\varphi}\left(\tau_{2}\right)$, and $h_{\varphi}(\tau)^{-1}=\varphi^{-1} \circ \tau^{-1} \circ \varphi$.

But $\varphi$ is connected to the identity map $1_{\mathrm{Y}}$ via a smooth path $s \mapsto \varphi_{s} \in \operatorname{Aut}(\mathrm{Y}, \lambda)$, defined on an open interval open interval $\mathscr{I}$ containing $[0,1]$, with $\varphi_{0}=1_{\mathrm{Y}}$ and $\varphi_{1}=\varphi$. That defines a smooth path of isomorphisms $h_{\varphi_{s}}=\varphi_{s} \circ \tau \circ \varphi_{s}^{-1}$. Let us denote $h_{s}$ for $h_{\varphi_{s}}$. The map $(s, t) \mapsto h_{s}(\operatorname{class}(t))$ is a plot defined on $\mathscr{I} \times \mathbf{R}$, in $\mathrm{T}_{\omega}$. By the monodromy theorem $\left[\mathrm{PIZ}_{13}, \$ 8.25\right]$, it has a global lifting $(s, t) \mapsto \mathrm{H}_{s}(t)$, defined on $\mathscr{I} \times \mathbf{R}$, which is a smooth plot in $\mathbf{R}$. And the lift is unique with $\mathrm{H}_{0}(0)=0$.

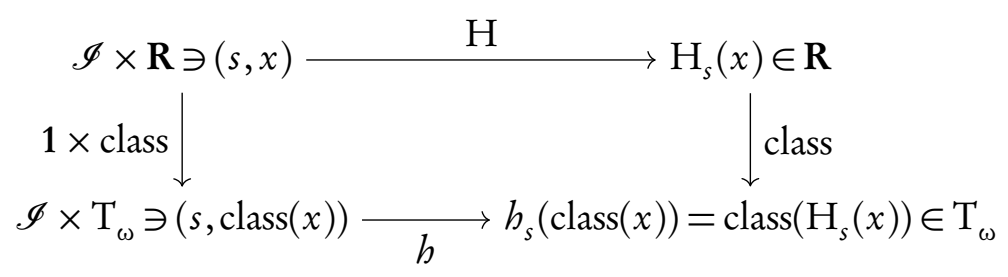

For every parameter $s$, the restriction $\mathrm{H}_{s}: \mathbf{R} \rightarrow \mathbf{R}$ is a smooth lifting of the isomorphism $h_{s}: \mathrm{T}_{\omega} \rightarrow \mathrm{T}_{\omega}$. Thus, up to a constant $b_{s} \in \mathbf{R}, \mathrm{H}_{s}$ is a smooth morphism from $\mathbf{R}$ to $\mathbf{R}$. Hence, $\mathrm{H}_{s}(x)=a_{s} x+b_{s}$. Where $s \mapsto a_{s}$ and $s \mapsto b_{s}$ are smooth, and $a_{s} \neq 0$ since $\mathrm{H}_{s}$ lifts an isomorphism. Now, for all $s \in \mathscr{I}$, all $x, x^{\prime} \in \mathbf{R}, h_{s}\left(\operatorname{class}\left(x+x^{\prime}\right)\right)=$ $h_{s}(\operatorname{class}(x))+h_{s}\left(\operatorname{class}\left(x^{\prime}\right)\right)$, that is, $\operatorname{class}\left(\mathrm{H}_{s}\left(x+x^{\prime}\right)\right)=\operatorname{class}\left(\mathrm{H}_{s}(x)\right)+\operatorname{class}\left(\mathrm{H}_{s}\left(x^{\prime}\right)\right)$, i.e. $a_{s}\left(x+x^{\prime}\right)+b_{s}=a_{s} x+b_{s}+a_{s} x^{\prime}+b_{s}+p$, and then $b_{s} \in \mathrm{P}_{\omega}$ for all $s \in \mathrm{P}_{\omega}$. 
Since $s \mapsto b_{s}$ is smooth and $\mathrm{P}_{\omega}$ is (diffeologically) discrete in $\mathbf{R}, b_{s}$ is constant and equal to $b_{0}$ which is 0 . Thus, $\mathrm{H}_{s}(x)=a_{s} x$. Next, $h_{s}(\operatorname{class}(x))=\operatorname{class}\left(\mathrm{H}_{s}(x)\right)$ implies that, for all $p \in \mathrm{P}_{\omega}$ there exists $p^{\prime} \in \mathrm{P}_{\omega}$ such that $\mathrm{H}_{s}(x+p)=\mathrm{H}_{s}(x)+p^{\prime}$. That is, $a_{s}(x+p)=a_{s} x+p^{\prime}$, and then $a_{s} p \in \mathrm{P}_{\omega}$ for all $p \in \mathrm{P}_{\omega}$. Again, since $\mathrm{P}_{\omega}$ is discrete in $\mathbf{R}, s \mapsto a_{s}$ is constant and the lifting $\mathrm{H}$ writes $\mathrm{H}_{s}(x)=a x$, for some number $a \neq 0$. Finally, since $\mathrm{H}_{0}$ lifts the identity $h_{0}=1_{\mathrm{T}_{\omega}}$ by a morphism, $\mathrm{H}_{0}(x)=x$ and $a=1$. Therefore $\mathrm{H}_{s}(x)=x$ for all $s$ and $h_{s}(\tau)=\tau$, that is, $\varphi \circ \tau_{\mathrm{Y}}=\tau_{\mathrm{Y}} \circ \varphi$, and the extension $\eta: \operatorname{Aut}(\mathrm{Y}, \lambda) \rightarrow \operatorname{Ham}(\mathrm{X}, \omega)$ is central.

(D) Let us show finally that $\eta: \operatorname{Aut}(\mathrm{Y}, \lambda) \rightarrow \operatorname{Ham}(\mathrm{X}, \omega)$ is surjective. Let $f \in \operatorname{Ham}(\mathrm{X}, \omega)$. There exists a smooth path $t \mapsto f_{t} \in \operatorname{Ham}(\mathrm{X}, \omega)$, such that $f_{0}=1_{\mathrm{X}}$ and $f_{1}=f$. We define, for all $x \in \mathrm{X}$, the path $\gamma_{\mathrm{X}}$ in $\mathrm{X}$ by $\gamma_{x}(t)=f_{t}(x)$. It satisfies $\gamma_{x}(0)=x$ and $\gamma_{x}(1)=f(x)$. The map $x \mapsto \gamma_{x}$ is smooth.

Given any $y \in \mathrm{Y}$ and $x=\pi(y) \in \mathrm{X}$, we will denote by $\underline{\gamma}_{y}$ the unique horizontal lifting of $\gamma_{x}$ with origin $y$. Moreover, the map $y \mapsto \underline{\gamma}_{y}$ is smooth and equivariant under the action of $\mathrm{T}_{\omega}\left[\mathrm{PIZ}_{13}, \$ 8.32\right]$. We define then:

$$
\left.\varphi(y)=\underline{\gamma}_{y}(1) \text { and } \varphi \in \mathrm{C}^{\infty}(\mathrm{Y}) \quad \text { (op. cit. }\right)
$$

The map $\varphi$ is a smooth lifting of $f$, that is, $\pi \circ \varphi=f \circ \pi$. Moreover, the equivariance of $\underline{\gamma}_{y}$ by $\mathrm{T}_{\omega}$ also implies that $\tau_{\mathrm{Y}} \circ \varphi=\varphi \circ \tau_{\mathrm{Y}}$ for all $\tau \in \mathrm{T}_{\omega}$. But if $\varphi$ is equivariant, it has no reason to preserve the contact form $\lambda$. We shall show then that there exists a map $\tau \in \mathrm{C}^{\infty}\left(\mathrm{Y}, \mathrm{T}_{\omega}\right)$ such that

$$
\Phi: y \mapsto \tau(y) \cdot \varphi(y)
$$

which is still a smooth lifting of $f$, preserves the contact form $\lambda$, that is, $\Phi \in \operatorname{Aut}(\mathrm{Y}, \lambda)$. Thanks to the partial derivatives formula (op. cit.), for any plot $r \mapsto y_{r}$ of $\mathrm{Y}$, we get:

$$
\begin{aligned}
\Phi^{*}(\lambda)\left(r \mapsto y_{r}\right) & =\lambda\left(r \mapsto \tau\left(y_{r}\right) \cdot \varphi\left(y_{r}\right)\right) \\
& =\theta\left(r \mapsto \tau\left(y_{r}\right)\right)+\lambda\left(r \mapsto \varphi\left(y_{r}\right)\right) \\
& =\tau^{*}(\theta)\left(r \mapsto y_{r}\right)+\varphi^{*}(\lambda)\left(r \mapsto y_{r}\right) .
\end{aligned}
$$

That is, $\Phi^{*}(\lambda)=\tau^{*}(\theta)+\varphi^{*}(\lambda)$. Consider now

$$
\Phi^{*}(\lambda)-\lambda=\tau^{*}(\theta)+\beta \quad \text { with } \quad \beta=\varphi^{*}(\lambda)-\lambda .
$$

Lemma I. The 1 -form $\beta$ is the pullback of a closed 1 -form $\varepsilon$ on $\mathrm{X}: \beta=\pi^{*}(\varepsilon)$.

4 Let us check that $\beta$ is closed: $d\left(\varphi^{*}(\lambda)-\lambda\right)=\varphi^{*}(d \lambda)-d \lambda=\varphi^{*}\left(\pi^{*} \omega\right)-\pi^{*} \omega=$ $\pi \circ \varphi)^{*} \omega-\pi^{*} \omega=(f \circ \pi)^{*} \omega-\pi^{*} \omega=\pi^{*} f^{*} \omega-\pi^{*} \omega=0$. Also, $\beta$ is invariant by $\mathrm{T}_{\omega}$ : $\tau^{*}(\beta)=\tau^{*}\left(\varphi^{*}(\lambda)-\lambda\right)=(\varphi \circ \tau)^{*}(\lambda)-\tau^{*}(\lambda)=(\tau \circ \varphi)^{*}(\lambda)-\lambda=\varphi^{*}\left(\tau^{*}(\lambda)\right)-\lambda=\varphi^{*}(\lambda)-\lambda=$ $\beta$. Moreover, $\beta$ vanishes vertically. Indeed, let us first remark that $\tau \circ \varphi=\varphi \circ \tau$, for all $\tau \in \mathrm{T}_{\omega}$, implies $\varphi \circ \hat{y}=\hat{y}^{\prime}$, for all $y \in \mathrm{Y}$ and $y^{\prime}=\varphi(y)$. Then, $\hat{y}^{*}(\beta)=\hat{y}^{*}\left(\varphi^{*}(\lambda)-\lambda\right)=$ $\hat{y}^{*}\left(\varphi^{*}(\lambda)-\hat{y}^{*}(\lambda)=(\varphi \circ \hat{y})^{*}(\lambda)-\hat{y}^{*}(\lambda)=\left(\hat{y}^{\prime}\right)^{*}(\lambda)-\hat{y}^{*}(\lambda)=\theta-\theta=0\right.$. Thus $\lambda^{\prime}=\lambda+\beta$ is 
a new connection 1 -form, the difference $\beta$ is then the pullback of a 1 -form on $\mathrm{X}$, according to $\left[\mathrm{PIZ}_{13}, \$ 8.37\right.$, Note].

Lemma 2. The 1-form $\varepsilon$ is exact: $\varepsilon=d \nu, \nu \in \mathrm{C}^{\infty}(\mathrm{X}, \mathbf{R})$.

Indeed, considering the fundamental property of the Chain-Homotopy Operator $\mathscr{K} \circ d+d \circ \mathscr{K}=\hat{1}^{*}-\hat{0}^{*}(\nabla)$, on the one hand, and the vanishing of the holonomy of the action of $\operatorname{Aut}(\mathrm{Y}, \lambda)$ on $\mathrm{Y}(\diamond)$ on the other hand, we get,

$$
0=\Psi_{\mathrm{X}}(\ell)=\hat{\ell}^{*}\left(\mathscr{K}_{\mathrm{X}}(\omega)\right)=\underline{\hat{\ell}}^{*}\left(\mathscr{K}_{\mathrm{Y}}(d \lambda)\right)=-\underline{\hat{\ell}}^{*}\left(d\left(\mathscr{K}_{\mathrm{Y}}(\lambda)\right)\right)=-d\left(\underline{\hat{\ell}}^{*}\left(\mathscr{K}_{\mathrm{Y}}(\lambda)\right)\right),
$$

for all $\ell \in \operatorname{Loops}(\mathrm{X})$ and all $\underline{\ell} \in \operatorname{Loops}(\mathrm{Y})$ over $\ell$, because $\hat{1} \circ \underline{\ell}^{*}=\hat{0} \circ \underline{\ell}^{*}$. Now, evaluating the Moment Map on the plot $t \mapsto \varphi_{t}$ connecting $1_{\mathrm{Y}}$ to $\varphi$, using $\bar{\ell}=\pi \circ \underline{\ell}$ and $\varphi_{t} \circ \underline{\ell}=\varphi_{t *}(\underline{\ell})$, we get:

$$
\begin{aligned}
d\left(\underline{\hat{\ell}}^{*}\left(\mathscr{K}_{\mathrm{Y}}(\lambda)\right)\left(t \mapsto \varphi_{t}\right)\right) & =d\left(\mathscr{K}_{\mathrm{Y}}(\lambda)\left(t \mapsto \varphi_{t *}(\underline{\ell})\right)\right)=d\left[t \mapsto \int_{\varphi_{t *}(\underline{\ell})} \lambda\right] \\
=\quad d\left[t \mapsto \int_{\underline{\ell}} \varphi_{t}^{*}(\lambda)\right] & =\int_{\underline{\ell}} \varphi^{*}(\lambda)-\int_{\underline{\ell}} \lambda=\int_{\underline{\ell}} \varphi^{*}(\lambda)-\lambda \\
=\quad \int_{\underline{\ell}}^{\beta} & =\int_{\underline{\ell}} \pi^{*} \varepsilon=\int_{\ell} \varepsilon .
\end{aligned}
$$

Thus, for all $\ell \in \operatorname{Loops}(\mathrm{X}), \int_{\ell} \varepsilon=0$. Therefore, according to [PIZI3, \$6.89], there exists $\nu \in \mathrm{C}^{\infty}(\mathrm{X}, \mathbf{R})$ such that $\varepsilon=d \nu$.

Now we can achieve to prove that $\Phi \in \operatorname{Aut}(\mathrm{Y}, \lambda)$. Indeed, let $\underline{\nu}=\nu \circ \pi \in \mathrm{C}^{\infty}(\mathrm{Y}, \mathbf{R})$. Let us define $\tau \in \mathrm{C}^{\infty}\left(\mathrm{Y}, \mathrm{T}_{\omega}\right)$ by $\tau=-$ class $\circ \underline{\nu}=-$ class $\circ \nu \circ \pi$, where class: $\mathrm{R} \rightarrow \mathrm{T}_{\omega}$. Hence:

$$
\tau^{*}(\theta)=-\pi^{*}\left(\nu^{*}\left(\operatorname{class}^{*}(\theta)\right)\right)=\pi^{*}\left(\nu^{*}(d t)\right)=-\pi^{*}(d \nu)=-\pi^{*}(\varepsilon)=-\beta .
$$

Thus $\tau^{*}(\theta)=-\varphi^{*}(\lambda)+\lambda$. Therefore:

$$
\Phi^{*}(\lambda)=\tau^{*}(\theta)+\varphi^{*}(\lambda)=-\varphi^{*}(\lambda)+\lambda+\varphi^{*}(\lambda)=\lambda, \quad \text { and } \quad \Phi \in \operatorname{Aut}(\mathrm{Y}, \lambda) .
$$

Until now, we have proved that $\eta: \operatorname{Aut}(\mathrm{Y}, \lambda) \rightarrow \operatorname{Ham}(\mathrm{X}, \omega)$ is surjective, we have to prove then that it is a subduction [PIZI3, \$1.46]. For this, we need to check that any plot $\mathrm{P}: r \mapsto f_{r}$ in $\operatorname{Ham}(\mathrm{X}, \omega)$, admits a local lifting $\tilde{\mathrm{P}}$ such that $\mathrm{P}={ }_{\text {locally }} \eta \circ \tilde{\mathrm{P}}$, everywhere. Thanks to the functional diffeology and to both subductions $\pi_{*}:$ Paths $(\mathrm{Y}) \rightarrow \operatorname{Paths}(\mathrm{X})$ $\left[\mathrm{PIZ}_{13}, \$ 8.32\right]$ and $\pi: \mathrm{Y} \rightarrow \mathrm{X}$, the map $(r, t, x) \mapsto f_{r, t}(x)$ is smooth and then admits a smooth lifting on $\mathrm{Y}$. Thus, for $x=\pi(y)$, the time $t=1$ of this lifting defines the smooth family $\varphi_{r}(y)$ of diffeomorphisms, the shift by $\tau \in \mathrm{C}^{\infty}\left(\mathrm{Y}, \mathrm{T}_{\omega}\right)$ preserves the smoothness of $r \mapsto \Phi_{r} \in \operatorname{Aut}(\mathrm{Y}, \lambda)$. 


\section{Moment Map of the Universal Extension Bundle Automorphisms}

In this section we will show how the sympletic manifold $(X, \omega)$ identifies, through the Moment map of the Hamiltonian action of $\operatorname{Aut}(\mathrm{Y}, \omega)$ with an orbit of this group for its linear coadjoint action on its space of momenta. We again emphazise the fact that this result generalizes the Kostant-Kirilov-Souriau theorem when the symplectic manifold is homogeneous under the action of a Lie group, and the symplectic form is integral. In the non-integral case but homogeneous, the optimal result in the category of manifolds states that the symplectic manifold is, up to a covering, an affine coadjoint orbit of the group. That result had been extended to the group of all Hamiltonian diffeomorphism in $[\mathrm{PIZI} 6]$.

6. Sympectic Manifolds As (Linear) Coadjoint Orbits - Let $(\mathrm{X}, \omega)$ be a symplectic manifold, and as it is described in (\$5), let $\mathrm{P}_{\omega}$ be its group of periods, $\pi: \mathrm{Y} \rightarrow \mathrm{X}$ be an integration bundle with connection $\lambda$, and $\operatorname{Aut}(Y, \lambda)$ be the group of automorphisms of the integration structure.

Let $\mathscr{A}^{*}$ be the space of Momenta of $\operatorname{Aut}(\mathrm{Y}, \lambda)$, that is, the space of left-invariant 1forms on $\operatorname{Aut}(Y, \lambda)$. The action of $\operatorname{Aut}(Y, \lambda)$ on $Y$ has a moment map, relatively to the parasymplectic form $d \lambda$, given by

$$
\mu_{\mathrm{Y}}: \rightarrow \mathscr{A}^{*}, \text { with } \mu_{\mathrm{Y}}(y)=\hat{y}^{*}(\lambda) \text {. }
$$

Then,

(I) The moment $\mu_{Y}$ projects on $\mu_{X}: X \rightarrow \mathscr{A}^{*}, \mu_{Y}=\mu_{X} \circ \pi$.

(2) $\mu_{Y}$ is equivariant under the coadjoint action of $\operatorname{Aut}(Y, \lambda)$.

(3) $\mu_{X}$ is injective.

(4) $\mu_{X}$ defines a diffeomorphism from $X$ onto the coadjoint orbit

$$
\mathscr{A}^{*} \supset \mathscr{O}_{\lambda}=\mu_{\mathrm{Y}}(\mathrm{Y})=\mu_{\mathrm{X}}(\mathrm{X}) \text {. }
$$

Therefore the symplectic manifold X inherits the structure of a coadjoint orbit. And this is a universal characterization of symplectic manifolds:

Every Symplectic Manifold is a (Linear) Coadjoint Orbit.

Which completes the statement made in [PIZi6] that Every Symplectic Manifold is a (Affine) Coadjoint Orbit of its group of Symplectomorphisms.

Proof. Let us begin by checking that $\mu_{\mathrm{Y}}$ is constant on each fiber. The action of $\mathrm{T}_{\omega}$ is central in $\operatorname{Aut}(\mathrm{Y}, \lambda)$, so for any $\tau \in \mathrm{T}_{\omega}$, for all $y \in \mathrm{Y}$ and for all $\varphi \in \operatorname{Aut}(\mathrm{Y}, \lambda)$ we have: $\widehat{\tau \cdot y}(\varphi)=\varphi(\tau \cdot y)=\tau \cdot \varphi(y)=\tau \cdot(\hat{y}(\varphi))$, hence $\widehat{\tau \cdot y}=\tau \circ \hat{y}$. Thus, $\mu_{\mathrm{Y}}(\tau \cdot y)=$ $(\widehat{\tau \cdot y})^{*}(\lambda)=(\tau \circ \hat{y})^{*}(\lambda)=\hat{y}^{*}\left(\tau^{*}(\lambda)\right)=\hat{y}^{*}(\lambda)=\mu_{Y}(y)$.

Now, let us denote, for all $\varphi, \psi$ in $\operatorname{Aut}(\mathrm{Y}, \lambda), \mathrm{R}(\varphi)(\psi)=\psi \circ \varphi^{-1}$, the right action of the group on its momenta. Then, the equivariance follows from: $\mu_{\mathrm{Y}}(\varphi(y))=\widehat{\varphi(y)}^{*}(\lambda)=$ 
$\left(\hat{y} \circ \mathrm{R}\left(\varphi^{-1}\right)^{*}(\lambda)=\mathrm{R}\left(\varphi^{-1}\right)^{*}\left(\hat{y}^{*}(\lambda)\right)=\mathrm{R}\left(\varphi^{-1}\right)^{*}\left(\mu_{\mathrm{Y}}(y)\right)=\mathrm{R}\left(\varphi^{-1}\right)^{*} \mathrm{~L}\left(\varphi^{-1}\right)^{*}\left(\mu_{\mathrm{Y}}(y)\right)=\right.$ $\operatorname{Ad}\left(\varphi^{-1}\right)^{*}\left(\mu_{\mathrm{Y}}(y)\right)=\operatorname{Ad}(\varphi)_{*}\left(\mu_{\mathrm{Y}}(y)\right)$.

Finally, pushing forward the moment maps [PIZI3, \$9.I2 \& 9.I3] leads to the commutative diagram below, where $\bar{\mu}_{\mathrm{X}}$ is the Moment Map for the group $\operatorname{Ham}(\mathrm{X}, \omega)$, and $\mathscr{H}^{*}$ denotes its space of momenta.

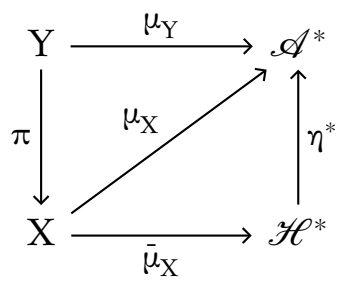

The Moment Map $\bar{\mu}_{X}$ is known to be injective [PIZI6], as well as $\eta^{*}$ since $\eta$ is a subduction (\$5, Proof $(\mathrm{C})$ ). Hence, $\mu_{\mathrm{X}}=\eta^{*} \circ \bar{\mu}_{\mathrm{X}}$ is injective and a subduction on $\mathscr{O}_{\lambda}=\mu_{\mathrm{Y}}(\mathrm{Y})$. Therefore, $\mu_{\mathrm{X}}$ is a diffeomorphism from $\mathrm{X}$ to $\mathscr{O}_{\lambda}=\mu_{\mathrm{Y}}(\mathrm{Y})$, equiped with the quotient diffeology of $\operatorname{Aut}(\mathrm{Y}, \lambda)$ by the stabilizer of any point $y \in \mathrm{Y}$.

\section{Conclusion}

This paper answers the question of the ontological nature of symplectic manifolds, if we can use such a big word. But that question has indeed arised in social networks, for example in mathoverfolw.net [Comr7]. That is a good justification a posteriori of this work.

As we have seen in this construction, for a symplectic manifold, to pass from an orbit of the affine action of the Hamiltonian diffeomorphisms, to an orbit of a linear action needs the integration of the Souriau's cocycle. This integration is done by considering the integration bundle of the symplectic manifold, which adds a floor to the construction $(\boldsymbol{\beta})$ and is summarized in the following diagram $(\boldsymbol{\phi})$. We have denoted by $G_{\lambda}$ the group of automorphisms of the integration structure, and $\pi_{\mathscr{O}}$ the subduction from $\mathrm{Y}$ onto its orbit, by quotient.

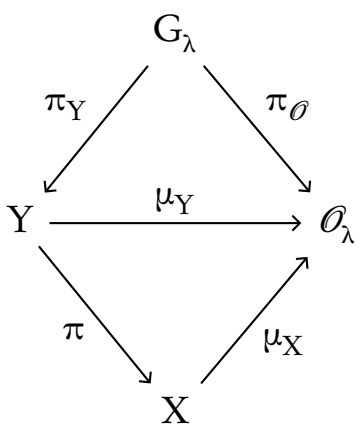

It is important to emphasize this, if the identification of the symplectic manifold as an affine-coadjoint orbit may possibly be adapted in another framework, dealing with infinite dimension spaces and groups, it seems not possible to avoid diffeology to transform the 
symplectic manifold into a linear-orbit of a group of diffeomorphisms. Indeed, the key of this construction being the integration bundle, there is no other known smooth theory for which it exists, except in the trivial case of an integral symplectic manifold, where it is a manifold. That is due to the dense nature of the group of periods of the symplectic form in general. Think for example to the simple case of the product $S^{2} \times S^{2}$, equiped with the symplectic form $\operatorname{Surf} \oplus \sqrt{2}$ Surf. Its group of periods $\mathbf{Z}+\sqrt{2} \mathbf{Z} \subset \mathbf{R}$ is dense and its torus of periods not Hausdorff, but indeed not diffeologically trivial.

\section{REFERENCES}

[Boo69] William M. Boothby. Transitivity of the automorphisms of certain geometric structures. Trans. Amer. Math. Soc. vol. 137, pp. 93-100, 1969.

[PDPI83] Paul Donato \& Patrick Iglesias. Exemple de groupes différentiels : flots irrationnels sur le tore, Preprint CPT-83/P.I524. Centre de Physique Théorique, Marseille, July 1983. http://math.huji.ac.il/ piz/documents/EDGDFISLT.pdf

[Don84] Paul Donato Revêtement et groupe fondamental des espaces différentiels homogènes. Thèse de doctorat d'état, Université de Provence, Marseille, 1984.

[Kos7o] Bertram Kostant. Orbits and quantization theory. In Actes, Congrès intern. Math., 1970. vol. 2, pp. 395-400.

[IZL9o] Patrick Iglesias and Gilles Lachaud. Espaces différentiables singuliers et corps de nombres algébriques. Ann. Inst. Fourier, Grenoble, 40(I):723-737, 1990.

[PIZ95] Patrick Iglesias-Zemmour. La Trilogie du Moment. Ann. Inst. Fourier, 45(3):825-857, (In French) 1995.

[PIZıo] The Moment Maps in diffeology. Memoirs of the American Mathematical Society, vol. 207, RI 2010.

[PIZI3] Diffeology, volume I85 of Mathematical surveys and monographs. American Mathematical Society, Providence, Rhode Island, 2013.

[PIZI6] _ Every symplectic manifold is a coadjoint orbit. Frontiers of Fundamental Physics I4 (FFPI4) - Mathematical Physics, vol/224, pp. I-17, September 2016. DOI: https://doi .org/10.22323/1.224.0141

[PIZI9] _ The moment maps in diffeology. Memoirs of the American Mathematical Society, vol. 207, RI 20IO. http://math . huji.ac.il/ piz/documents/DBlog-EX-DOHFTB.pdf

[Kir74] Alexandre A. Kirillov. Elements de la théorie des représentations. Ed. MIR, Moscou, 1974.

[MW82] Jerry Marsden and Alan Weinstein. The hamiltonian structure of the maxwell-vlasov equations. Physica D, 4:394-406, 1982.

[Omo86] Stephen Malvern Omohundro. Geometric Perturbation Theory in Physics. World Scientific, 1986.

[Sou7o] Jean-Marie Souriau. Structure des systèmes dynamiques. Dunod Ed., Paris, 1970.

[Comı] https://mathoverflow.net/questions/147395/

Paul Donato, Institut de Mathématiques de Marseille, Aix-Marseille Université

E-mail address: paul.donato@univ-amu.fr

Patrick Iglesias-Zemmour, The Hebrew University of Jerusalem, Israel.

E-mail address: piz@math.huji.ac.il

URL: http://math.huji.ac.il/ piz 